\title{
Semantic-based technologies for video analysis in activity recognition, video surveillance and smart home domains
}

\author{
Pierluigi Ritrovato $^{1} \cdot$ Luca Greco $^{1}$
}

Published online: 30 June 2020

○) Springer-Verlag GmbH Germany, part of Springer Nature 2020

Miniaturisation of video recording devices, amazing computing capabilities provided by embedded systems processors, worldwide explosion of smart IP cameras with more than 300 millions devices on the planet generating $65 \%$ of universal big data, are moving video analysis among the hottest research topics of the last years. Even the industrial sector recognise the relevance of Video analysis. Several Gartner Hype Cycle reports (on Analytics and Business Intelligence and on Data Science and Machine Learning) put video and image analysis in the "climbing the scope" position, so close to become part of commercial solutions in less than 2 years, leading to the application of research results in several industrial domains like video surveillance, human activity recognition, robot control and smart environments, just to mention few. This increasing interest are feeding the development of new video analysis approaches that move away from the traditional pattern matching and statistical models that start from low level and local feature information for give a meaning to the element in the scene. The enormous development that are achieving the Artificial Intelligence and in particular knowledge management and Semantic Web movement both in terms of research and technology, influenced many research areas including the image and video analysis. The development of these technologies are paving the way to the development of cognitive approaches that propose building a symbolic model of the world, expressed in a logic-based language, which abstractly represents the scene objects and their relations. Indeed, in the last 5 years we assisted to the proposition of hybrid approaches where results produced by traditional bottom-up feature-based vision algorithms are fused with top-down approach based on semantic web technologies

Pierluigi Ritrovato

pritrovato@unisa.it

Luca Greco

lgreco@unisa.it

1 University of Salerno, Salerno, Italy where typically context/domains information are described using ontologies and decision (interpretation of raw-data) are taken using rule based systems. These approaches seems to be promising not only for object tracking in video surveillance systems but also for human activity and action recognition in Ambient Intelligence and Smart Home environment, image analysis and video analytics.

The special issue includes six papers, three of them, centered on Ambient Assisted Living, show the effectiveness of semantic web technologies for developing advanced systems where low level information (coming from sensors) can be fused with computer vision techniques for activity recognition, with relevant improvement with respect to traditional approaches. The other three papers demonstrate the effectiveness of building solutions following an hybrid approach combining knowledge based techniques with traditional ones in context like object tracking and image analysis.

Enjoy your read!

Guest Editors:

Prof. Pierluigi Ritrovato: DIEM Department of Information and Electrical Engineering and Applied mathematics

Pierluigi Ritrovato received the master degree in computer science in 1992 and from 2016 is Associate Professor in "Information Processing Systems" at the University of Salerno teaching courses of Internet of Things and Semantic Enterprise Systems at the bachelor and master degree in Computer Engineering. In 2014, he joined to the MIVIA (Intelligent Machine for Video, Image and Audio processing) laboratory leading research in the area of Cognitive Vision. In the last 10 years, he has been focusing his scientific research on Knowledge Management, Semantic Web, Adaptive Learning Management Systems, Grid middleware for Service Oriented Architecture. He has been scientific coordinator of several research projects in the area of knowledge management and intelligent adaptive systems funded by the European Commission. 
Prof. Luca Greco: DIEM Department of Information and Electrical Engineering and Applied mathematics

Luca Greco is an Assistant Professor and member of the Artificial Vision Research Group at the University of Salerno. He received in 2008 the Laurea Master degree (cum laude) in Electronic Engineering, and in 2013 the Ph.D. in Electronic and Computer Engineering, both from the University of Salerno, Italy. His interests cover the areas of image and video analysis, machine learning and semantic technologies with applications like intelligent video surveillance, information retrieval and sentiment analysis. He authored more than 40 research papers in international journals, conference proceedings and book chapters.

Publisher's Note Springer Nature remains neutral with regard to jurisdictional claims in published maps and institutional affiliations. 